\section{ALGUNS CONCEITOS DA SOCIOLOGIA DE PIERRE BOURDIEU RELACIONADOS COM A ANÁLISE DE REDE SOCIAL'}

\author{
Bruno Henrique Alves* \\ Ely Francina Tannuri Oliveira**
}

RESUMO O Campo da Ciência da Informação $(\mathrm{Cl})$ é um espaço hierarquizado, relativamente autônomo e formado por relações objetivas que se dão em um contexto social. Esta pesquisa objetiva compreender os mecanismos implícitos à geração e construção do conhecimento dos pesquisadores bolsistas de produtividade em pesquisa do CNPq - Conselho Nacional de Desenvolvimento Científico e Tecnológico - em Cl e a posição social que ocupam no Campo em questão. De forma mais específica, pretende: compreender os mecanismos sociais construídos para organizar e facilitar a estruturação das redes de colaboração científica dos pesquisadores bolsistas de produtividade em pesquisa do $\mathrm{CNPq}$ em Cl; e fundamentar a Análise de Redes Sociais - ARS, a partir da teoria do Campo e os conceitos de Capital Social e Capital Científico, propostos pela Sociologia de Pierre Bourdieu. Para a construção das redes de colaboração científica entre os pesquisadores, utilizou-se o Software Ucinet. A teoria do Campo Científico foi utilizada também para complementar o procedimento de Análise de Redes Sociais, com foco na caracterização e evolução das redes de colaboração científica (2005-2009 e 2010-20 I4). Como conclusões, destaca-se que, de acordo com os fundamentos da Sociologia de Pierre Bourdieu, para ocupar uma posição de dominante, o pesquisador bolsista de produtividade em pesquisa do CNPq deve entender a lógica do jogo, pois o Campo possui eixos estruturantes a partir dois quais se identificam os principais jogadores que ocupam esse espaço social de concorrência científica.

Palavras-chave: Sociologia de Pierre Bourdieu. Análise de Rede Social. Ciência da Informação.
* Doutor em Ciência da Informação pela Universidade Estadual Paulista "Júlio de Mesquita Filho", Brasil. Professor do Departamento de Ciência da Informação da Universidade Federal Fluminense, Brasil.

E-mail: brhenriquealves@gmail.com.

** Livre-Docente na área de Estudos Bibliométricos pela Universidade Estadual Paulista Júlio de Mesquita Filho, Brasil. Doutora em Educação pela Universidade Estadual Paulista Júlio de Mesquita Filho, Brasil.. Coordenadora do Grupo de Pesquisa Estudos métricos da Informação.

E-mail: etannuri@flash.tv.br

\section{INTRODUÇÃO}

P ara se compreender o processo e as características que interferem e contribuem 1 para a construção do conhecimento científico em determinado Campo, destacam-se as contribuições da Sociologia da Ciência - "que estuda os modos pelos quais a pesquisa científica e a

I Baseado na tese "Sociologia de Pierre Bourdieu e os pesquisadores bolsistas de produtividade em pesquisa do CNPq em Ciência da Informação", com financiamento do Conselho Nacional de Desenvolvimento Científico e Tecnológico (CNPq). difusão do conhecimento científico são influenciadas pelas condições sociais e, por seu turno, influenciam o comportamento social" (BEN-DAVID, 1975, p.1) -, que utilizam a própria ciência como objeto de estudo, pois investigam as relações entre as pessoas em um determinado espaço buscando consigná-lo como Campo de poder (BOURDIEU, 2008).

O conjunto de conceitos da Sociologia de Pierre Bourdieu contribui para o desenvolvimento da Ciência da Informação (CI) e seu fortalecimento enquanto Campo, considerando os aspectos interdisciplinares da $\mathrm{CI}$, que recebe aportes da Biblioteconomia, 
Ciência da Computação e Sociologia da Ciência, entre outros (PINHEIRO, 1997).

A partir dessas assertivas, surgem algumas questões: como esses pesquisadores se colocam no Campo da CI e qual a posição que ocupam no campo em estudo? Como explicar os mecanismos que fazem com que os pesquisadores passem a ocupar posições de dominantes, dominados e pretendentes no Campo da CI?

Esta pesquisa objetiva, de forma geral, compreender os mecanismos implícitos à geração e construção do conhecimento dos pesquisadores bolsistas de produtividade em pesquisa do Conselho Nacional de Desenvolvimento Científico e Tecnológico - CNPq em CI, a produção científica publicada, as redes de colaboração científica que se formam a partir das relações sociais para assegurar a produção do conhecimento e a posição social que ocupam no Campo da CI.

De forma específica, propõe-se compreender os mecanismos sociais construídos para organizar e otimizar a estruturação das redes de colaboração científica dos pesquisadores bolsistas de produtividade em pesquisa do $\mathrm{CNPq}$ em CI, bem como fundamentar a Análise de Redes Sociais - ARS, a partir da teoria do Campo e dos conceitos de Capital Social e Capital Científico, propostos pela Sociologia de Pierre Bourdieu.

Nesse sentido, esta pesquisa justifica-se em virtude de serem pouco explorados os estudos que objetivam compreender a CI como um Campo Científico, a partir da produção científica dos pesquisadores bolsistas de produtividade em pesquisa do $\mathrm{CNPq}$ em $\mathrm{CI}$, e de explicar as estruturas e a formação das redes de colaboração científica utilizando os conceitos da Sociologia de Pierre Bourdieu.

\section{REFERENCIAL TEÓRICO}

\section{I O campo científico}

Para Bourdieu (1983, 2004a), todo Campo, inclusive o Campo Científico, é um espaço de forças e lutas que possui regras próprias. Nesse espaço, sempre há lutas, competição e disputas por troféus, de acordo com os interesses próprios de cada Campo.

A estrutura do Campo Científico é um estado da relação de força entre os diferentes agentes e/ou instituições engajadas na luta científica composta pela distribuição do Capital Científico por aqueles inseridos no mesmo. Essa luta possui uma lógica que só é compreendida por quem está participando do jogo, pois os jogadores disputam entre si as posições e poder para serem utilizados em seu favor.

Para Bourdieu (2004b, 2008), o conceito de Capital Científico está classificado em duas espécies: o "puro, específico ou autoridade propriamente científica" e o "institucional, temporal, político ou poder sobre o mundo científico ". O primeiro está relacionado ao poder específico, 'prestígio' pessoal, que consiste no reconhecimento junto aos seus pares em determinado Campo Social. Esse reconhecimento aumenta na medida em que o pesquisador e/ou intelectual obtém sucesso na atividade e descoberta científica, que contribui diretamente para o desenvolvimento da sociedade e do seu espaço social.

O poder institucional e institucionalizado está ligado à ocupação de posições importantes nas instituições científicas e/ou administração científica, tais como coordenador de grupo de pesquisa, chefe de departamento, comitês de avaliação, entre outros. Esse tipo de Capital é acumulado a partir de estratégias que cada agente e/ou instituição adota para ocupar uma posição de destaque no interior do Campo Científico. Para aumentar esse tipo de Capital, é preciso adotar estratégias "políticas" de acordo com as dinâmicas do Campo, por exemplo, no Campo Científico, pertencer à uma banca de defesa, participação em "[...] congressos, formação acadêmica em instituições de maior prestígio ou mesmo a quantificação das publicações, não devendo ser confundidas com as mesmas estratégias do campo político» (MOREIRA JR.; ANDRADE, 2014, p.173).

Esse Capital Científico pode ser representado também como Capital Científico do tipo Objetivado, considerando os aspectos teóricos do Capital Cultural, que pode ser representado pelo indicador de produção, indicando o número de documentos publicados por um ou mais agentes e/ou instituição.

\subsection{Capital social}

O Capital Social é o conjunto de recursos reais ou potenciais ligados à posse de uma rede durável de relações mais ou menos 
institucionalizadas de interconhecimento e interreconhecimento. Em outras palavras, à vinculação a um grupo, como um conjunto de agentes que não são apenas dotados de propriedades comuns (susceptível de serem percebidas pelo observador, por outras pessoas ou por elas próprias), mas também estão unidos por ligações permanentes e úteis. O volume de Capital Social possuído por um agente depende da extensão da rede de conexões que ele pode, efetivamente, mobilizar e sobre o volume de Capital Econômico, Cultural, Científico ou Simbólico que ele possui e ao qual está vinculado (BOURDIEU, 1980).

O Capital Social corresponde ao conjunto de relações sociais, que compreende o relacionamento e a rede de contatos que determinado agente possui no interior de um Campo Científico, considerando as posições de dominantes, dominados e pretendentes que se apresentam em determinada estrutura social. O Capital Social na ARS pode ser representado, por exemplo, a partir da interação social entre os indivíduos inseridos nos diferentes contextos - nacional e/ou internacional -, apresentando, principalmente, aspectos científicos, sociais, culturais e tecnológicos. Essa interação social pode ser visualizada por meio de uma rede de colaboração científica, que possibilita aprofundar a análise de sua estrutura por meio de indicadores de rede, com destaque, neste caso, para o grau de centralidade (centrality degree).

Considerando os aspectos apresentados nesta pesquisa e seu objeto de estudo, o Capital Social pode ser operacionalizado a depender do ponto de vista do pesquisador; seu valor para a investigação em CI proporciona um enquadramento para compreender a relação entre estrutura social e o compartilhamento de informação e conhecimento entre os agentes em um espaço social, no qual se apresentam conflitos e concorrências científicas (JOHNSON et al., 2008).

\subsection{Análise de rede social para visualização da rede de colaboração científica}

Uma das aplicações mais comuns das ARS é o estudo da formação e estrutura das redes de colaboração científica. Essa análise possibilita identificar as relações entre os diferentes agentes em determinado Campo Científico por meio da autoria científica (HAYASHI et al., 2012).
A formação das redes sociais é o resultado de um processo dinâmico, pois elas evoluem ao longo do tempo e no espaço social. As propriedades e estrutura social de uma rede evoluem e se modificam no contexto histórico e social, considerando que sua composição e constituição são influenciadas por fatores econômicos, sociais e políticos. As redes sociais não só possuem propriedades topológicas/ estruturais, mas características dinâmicas (NEWMAN; BARABÁSI; WATTS, 2006). A dinâmica da rede surge como possibilidade de pesquisa quando se pretende compreender "o processo que ocorre quando as conexões e atores surgem ou desaparecem da rede, indicando a maneira que a estrutura será alterada ao longo do tempo" (MARTINS, 2012, p.23).

A literatura apresenta alguns indicadores, a fim de aprofundar a análise da estrutura de uma rede. Alguns deles, como densidade - density - e medidas de centralidade, são descritos a seguir.

A densidade (density) permite avaliar a coesão e a estrutura da rede, e é calculada pela razão entre o número de laços (ligações, conexões e/ou relacionamentos) existentes na rede pelo número total de laços possíveis (OTTE; ROUSSEAU, 2002).

O grau de centralidade (centrality degree) de um ator é o número de atores na rede com quem ele se relaciona ou está conectado em pelo menos um artigo, ou seja, é o número de ligações que um ator tem com todos os demais atores.

Para Otte e Rousseau (2002), a centralidade de proximidade (closenness centrality) é uma medida inversa da centralidade, em que um valor maior indica um ator menos central, enquanto um valor menor indica um ator mais central.

A centralidade de intermediação (betweenness centrality) pode ser definida como o número de vezes que um nó intermedeia um caminho mais curto (geodésico) entre outros dois nós da rede.

Em termos de Sociologia de Pierre Bourdieu, os indicadores apresentados - densidade (density), grau de centralidade (centrality degree), centralidade de proximidade (closenness centrality) e centralidade de intermediação (betweenness centrality) - podem estar representados em determinado Campo Científico, no caso, a CI, de forma a compreender a estrutura social desse espaço, que é formado por dominantes, dominados e pretendentes. Os pesquisadores bolsistas de produtividade 
em pesquisa do CNPq no Campo da CI, que possuem destaque nas medidas citadas, podem ser considerados dominantes de uma determinada rede de colaboração, considerando que possuem uma posição significativa diante de seus "concorrentes" nesse Campo de relações objetivas, buscando o acúmulo de Capital Científico e Capital Social.

\section{PROCEDIMENTOS METODOLÓGICOS}

Para a construção da rede de colaboração científica entre os pesquisadores, no primeiro quinquênio (2005-2009), utilizou-se o Software Ucinet. Foram considerados os pesquisadores enquadrados no nível/categoria 1A, 1B, 1C, 1D e 2, que publicaram em coautoria, a partir dos 317 artigos científicos: 36 pesquisadores bolsistas de produtividade em pesquisa do $\mathrm{CNPq}$ em $\mathrm{CI}$ que publicaram em coautoria e 7 pesquisadores estrangeiros e seus coautores doutores, num total de 104 pesquisadores na rede de colaboração científica. Foram utilizados 88 artigos em coautorias com doutores, em âmbito nacional e/ ou internacional.

Para a construção da rede de colaboração científica entre os pesquisadores, no segundo quinquênio (2010-2014), utilizou-se o Software Ucinet. Foram considerados os pesquisadores enquadrados no nível/categoria SR, 1A, 1B, 1C, $1 \mathrm{D}$ e 2, que publicaram em coautoria, a partir dos 597 artigos científicos: 48 pesquisadores bolsistas de produtividade em pesquisa do $\mathrm{CNPq}$ em CI que publicaram em coautoria e 28 pesquisadores estrangeiros e seus coautores doutores, totalizando 228 pesquisadores na rede. Foram utilizados 259 artigos em coautorias com doutores, em âmbito nacional e/ou internacional.

Para a construção das redes de colaboração científica, no primeiro (2005-2009) e segundo quinquênios (2010-2014) em estudo, verificou-se $\mathrm{o}$ ano em que os pesquisadores obtiveram o título de doutor, pois foram selecionados como doutores aqueles que publicaram depois e no período em que defenderam a tese, considerando a data da defesa.

\section{I Análise de redes sociais (ARS) e a sociologia de Pierre Bourdieu}

A teoria do Campo Científico foi utilizada como fundamento teórico para a interpretação da ARS, focando a identificação, caracterização e evolução das redes de colaboração científica (2005-2009 e 2010-2014). Estes aspectos são relacionados com os conceitos de Capital Científico e Capital Social, propostos pela Sociologia de Pierre Bourdieu, tendo em vista identificar a posição de dominantes, dominados e pretendentes na estrutura social do Campo da CI. São conceitos traduzidos e interpretados no contexto das redes de colaboração científica (BOURDIEU; CHAMBOREDON; PASSERON, 2015).

Dessa forma, relacionam-se os indicadores de rede - grau de centralidade e centralidade de intermediação - com os conceitos propostos pela Sociologia de Pierre Bourdieu para compreender a estrutura social na qual estão inseridas as relações sociais entre os pesquisadores bolsistas de produtividade em pesquisa do $\mathrm{CNPq}$ e seus coautores doutores no Campo Científico da CI.

\section{APRESENTAÇÃo E ANÁLISE DOS DADOS}

Os dados são apresentados e analisados de acordo com os objetivos, de modo a: compreender os mecanismos sociais construídos para organizar e otimizar a estruturação das redes de colaboração científica dos pesquisadores bolsistas de produtividade em pesquisa do $\mathrm{CNPq}$ em CI; e fundamentar a ARS, a partir da teoria do Campo, e os conceitos de Capital Social e Capital Científico, propostos pela Sociologia de Pierre Bourdieu.

\section{I Rede de colaboração científica, no período de 2005 a 2009}

$\mathrm{Na}$ análise da rede de colaboração científica, representada no Grafo 1, os triângulos em verde representam 36 pesquisadores bolsistas de produtividade em pesquisa do $\mathrm{CNPq}$ em CI (2005-2009); os quadriláteros em vermelho representam os pesquisadores estrangeiros (sete); e os círculos em azul, os pesquisadores brasileiros que possuem o título de doutor (61). Destaca-se que a espessura dos segmentos de retas é proporcional à intensidade de coautoria entre eles. 
Grafo 1: Rede de colaboração científica (2005-2009).

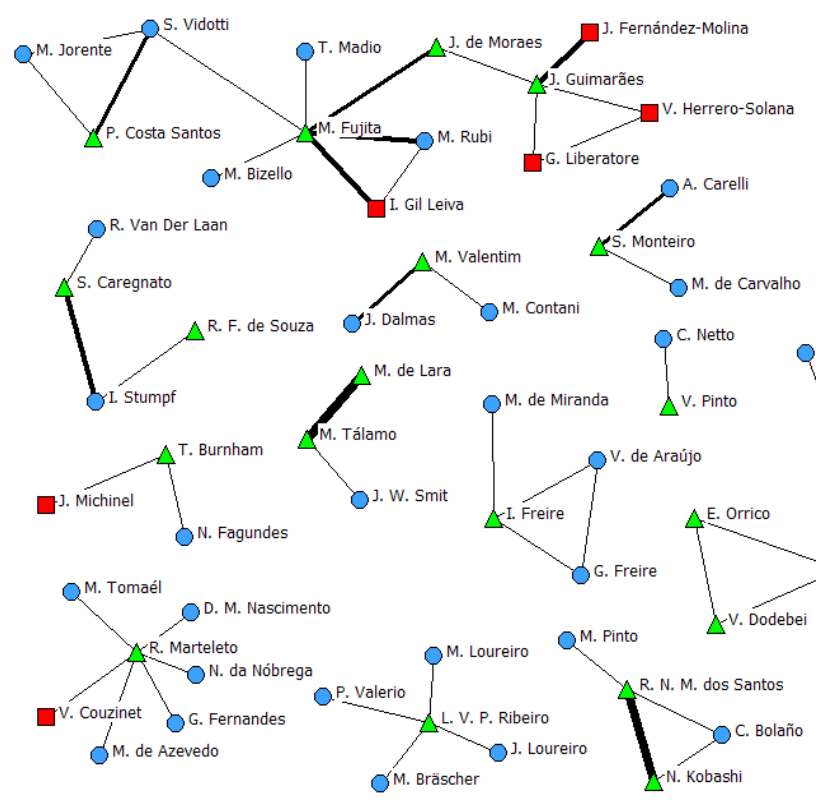

Fonte: Elaborado pelos autores por meio do Software Ucinet.
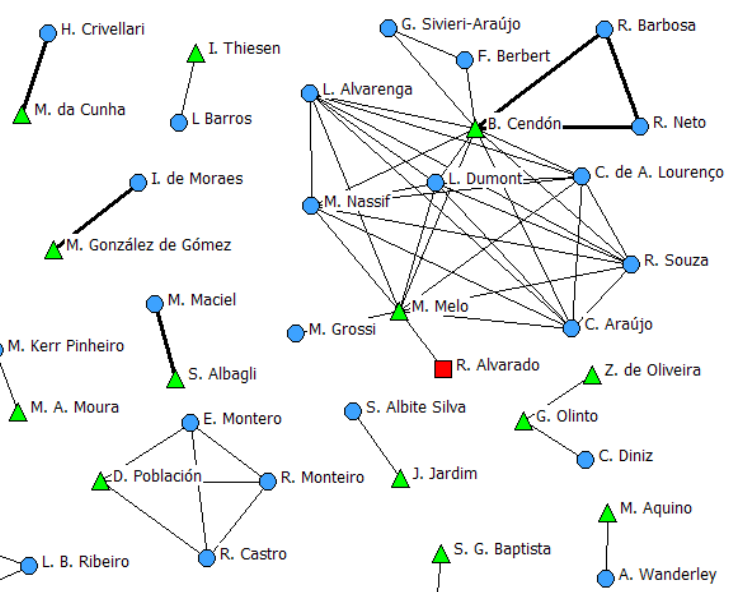

$\triangle$ J. Jardim

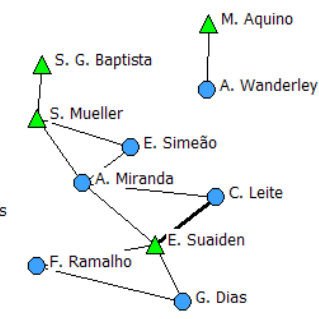

Legenda:

$\triangle$ Pesquisadores PQ OPesquisadores doutores Pesquisadores estrangeiros
A análise do Grafo 1 mostra um grande subcampo, na parte superior e esquerda, formado a partir de conexões com outros subcampos que se comunicam entre si, mostrando a formação de uma rede de colaboração científica que inclui quatro pesquisadores bolsistas PQ em CI - P. Costa Santos, M. Fujita, J. de Moraes e J. Guimarães - e nove coautores doutores, sendo quatro estrangeiros: I. Gil Leiva (Universidad de Extremadura-Espanha), G. Liberatore (Universidad Nacional de Mar del PlataArgentina), J. Fernández-Molina (Universidad de Granada-Espanha) e V. Herrero-Solana (Universidad de Granada-Espanha). Isso mostra a interlocução brasileira no contexto internacional, bem como a visibilidade científica e sua inserção no contexto mundial para contribuir com a construção do conhecimento científico, especialmente no interior do Campo da CI.

\subsection{Rede de colaboração científica, no período de 2010 a 2014}

$\mathrm{Na}$ análise da rede de colaboração científica entre os pesquisadores bolsistas de produtividade em pesquisa do $\mathrm{CNPq}$ em CI e seus coautores doutores em âmbito nacional e internacional, representada no Grafo 2, verificase que: os triângulos em verde representam os pesquisadores bolsistas de produtividade em pesquisa do CNPq em CI (48); os círculos em azul, seus coautores, que pertencem ao grupo de 152 doutores em âmbito nacional; e os 28 pesquisadores, quadriláteros em vermelho, são estrangeiros, a saber: Alan Gilchrist (Consultor, The Cura Consortium, Reino Unido); Carlos G. F. Paniagua (Universidad de Salamanca-Espanha); Chaim Zins (University of Haifa-Israel); Chun Wei Choo (University of Toronto-Canadá); Daniel Martínez-Avila (Espanha-Unesp-Marília); Douglas Tudhope (University of South WalesReino Unido); Janet Harrison (Loughborough University, Reino Unido); Javier Noguera (Universidad de Zaragoza-Espanha); Juan Carlos Fernández-Molina (Universidad de GranadaEspanha); María del Carmen Agustín Lacruz (Universidad de Zaragoza-Espanha).

Em relação aos pesquisadores estrangeiros - Maria Isabel Escalona (Espanha-Universidad de Extremadura); Maria Laura Martinez (UruguaiUniversidade de São Paulo); Maria Luisa Conde Villaverde (Espanha-Dirección de Archivos 
Estatales); Raquel Gómez-Díaz (Universidad de Salamanca-Espanha); Susan Kovacs (Université de Lille 3-França); Yolande Maury (Université de Lille 3-França); Francisco J. García Marco (Universidad de Zaragoza-Espanha); Antonia Salvador Benitez (Universidad Complutense de Madrid-Espanha); Daniel Barredo Ibáñez (Universidad Laica Eloy Alfaro de ManabíEquador); Antonio Pulgarin Guerrero (Universidad de Extremadura-Espanha); Aurora Cuevas-Cerveró (Universidad de GranadaEspanha); José Antonio Moreiro González
(Universidad Carlos III de Madrid-Espanha); Manuel Valente Mangue (MoçambiqueUniversidade Eduardo Mondlane); Ana Lúcia Silva Terra (França-Universidade do Porto); Isidoro Gil Leiva (Universidad de Murcia-Espanha); María Gladys Ceretta-Soria (Universidad de la República-Uruguai); Viviane Couzinet (Université Toulouse III Paul SabatierFrança); Gustavo Liberatore (Universidad Nacional de Mar del Plata-Argentina) -, destacase que alguns possuem afiliação estrangeira e atuam em universidades brasileiras.

Grafo 2: Rede de colaboração científica (2010-2014)

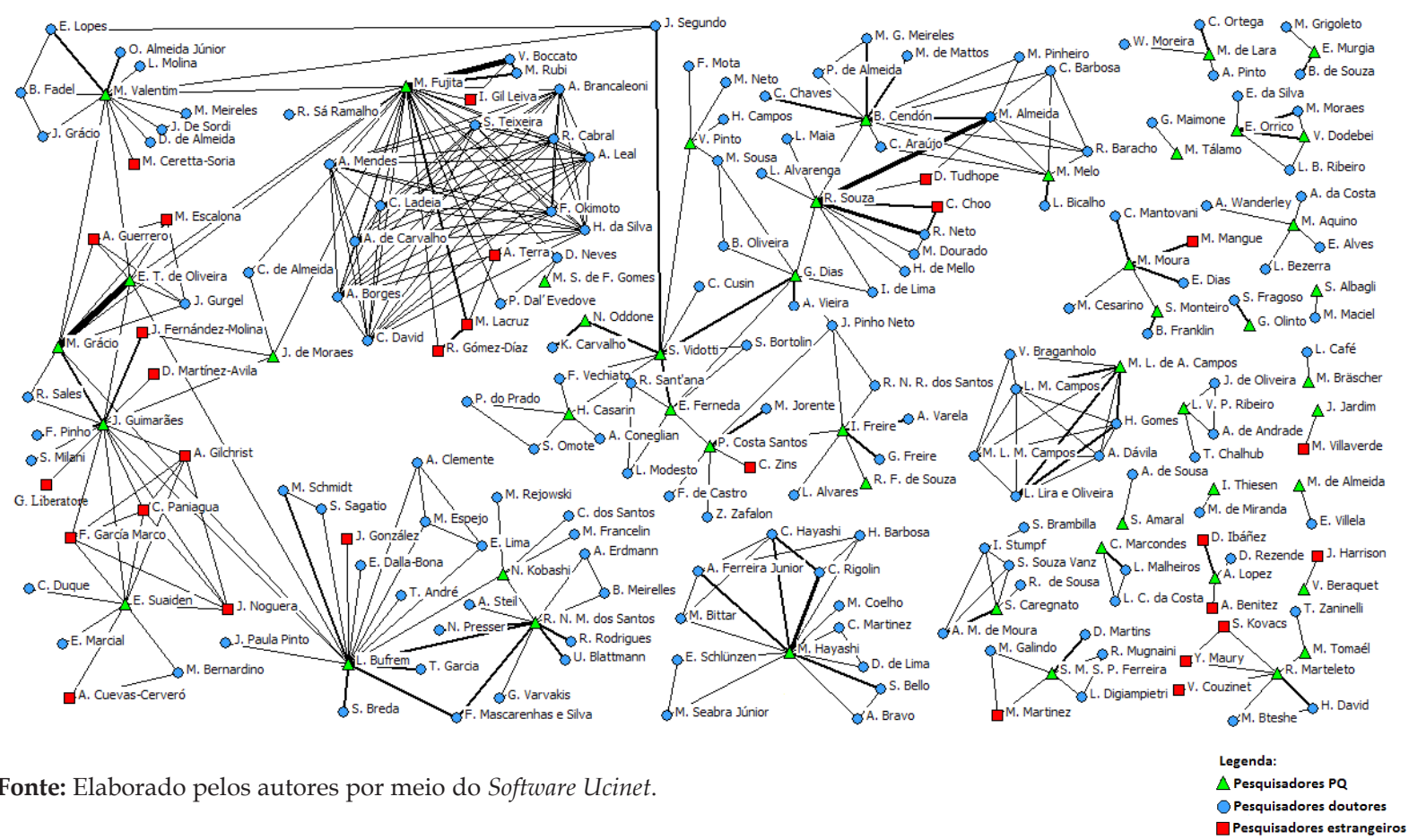

No Grafo 2, as espessuras dos segmentos de retas são proporcionais à intensidade de coautorias entre os pesquisadores bolsistas de produtividade em pesquisa do $\mathrm{CNPq}$ em $\mathrm{CI}$ e seus coautores doutores em âmbito nacional e internacional. Não estão representados na rede os seguintes pesquisadores bolsistas de produtividade em pesquisa do $\mathrm{CNPq}$ em $\mathrm{CI}$ : Aldo de Albuquerque Barreto, Jaime Robredo, Lídia Silva de Freitas, Liz Rejane Issberner, Maria Nelida González de Gomez, Miriam Figueiredo Vieira da Cunha, Rubens Ribeiro Gonçalves da Silva e Suzana Pinheiro Machado Mueller, considerando que não publicaram artigos científicos no período em estudo e/ou não apresentaram coautorias.

A análise do Grafo 2 mostra, à esquerda e na parte superior, um grande subcampo formado a partir de conexões com outros subcampos que se comunicam entre si, mostrando a formação de uma rede de colaboração científica, que inclui 23 pesquisadores bolsistas de produtividade em pesquisa do $\mathrm{CNPq}$ em $\mathrm{CI}$, a saber: M. Valentim, M. Fujita, E. T. de Oliveira, M. Grácio, J. Guimarães, E. Suaiden, J. de Moraes, L. Bufrem, N. Kobashi, M. S. de F. Gomes, N. Oddone, 
H. Casarin, R. N. M. dos Santos, S. Vidotti, E. Ferneda, P. Costa Santos, I. Freire, R. F. de Souza, G. Dias, R. Souza, V. Pinto, B. Cendón, M. Melo, com 112 coautores, sendo 19 estrangeiros. Isso mostra a interlocução brasileira no contexto internacional, bem como a visibilidade científica e sua inserção no contexto mundial para contribuir na construção do conhecimento científico, especialmente no interior do Campo da CI.

\subsection{Indicadores da rede de colaboração científica, no período de 2005 a 2009}

Apresentam-se, na Tabela 1, os diferentes graus de centralidade (centrality degree), destacando-se a pesquisadora B. Cendón com maior grau de centralidade - 11.0 -, significando que possui 11 ligações ou coautorias com os demais pesquisadores.

M. Melo, pesquisadora bolsista de produtividade em pesquisa do $\mathrm{CNPq}$ em CI, possui o segundo maior grau de centralidade nove -, significando que possui nove ligações ou coautorias com os demais pesquisadores.

Tabela 1: Cálculo do grau de centralidade dos pesquisadores bolsistas de produtividade em pesquisa do $\mathrm{CNPq}$ em Ciência da Informação (2005-2009)

\begin{tabular}{|c|c|}
\hline Pesquisadores & Degree \\
\hline B. Cendón & 11.0 \\
\hline M. Melo & 9.0 \\
\hline M. Fujita & 6.0 \\
\hline R. Marteleto & 6.0 \\
\hline M. L. de A. Campos & 5.0 \\
\hline E. Suaiden & 4.0 \\
\hline J. Guimarães & 4.0 \\
\hline L. V. P. Ribeiro & 4.0 \\
\hline D. Población & 3.0 \\
\hline I. Freire & 3.0 \\
\hline R. N. M. dos Santos & 3.0 \\
\hline S. Mueller & 3.0 \\
\hline C. Marcondes & 2.0 \\
\hline E. Orrico & 2.0 \\
\hline G. Olinto & 2.0 \\
\hline J. de Moraes & 2.0 \\
\hline M. Tálamo & 2.0 \\
\hline M. Valentim & 2.0 \\
\hline
\end{tabular}

N. Kobashi

P. Costa Santos

2.0

S. Caregnato

2.0

S. Monteiro

2.0

T. Burnham

2.0

V. Dodebei

2.0

I. Thiesen

2.0

J. Jardim

1.0

M. A. Moura

1.0

M. Aquino

M. da Cunha

1.0

1.0

M. de Lara

M. González de Gómez

1.0

1.0

R. F. de Souza

1.0

S. Albagli

S. G. Baptista

V. Pinto

Z. de Oliveira

Fonte: Dados calculados pelo Software Ucinet.

Observando o grau de centralidade dos pesquisadores bolsistas de produtividade em pesquisa do CNPq em CI (2005-2009), o fenômeno constatado reforça a afirmação de que alguns deles compõem o grupo de pesquisadores centrais ou periféricos da rede de colaboração científica. Apresentam-se, na Tabela 2, as diferentes centralidades de intermediação dos pesquisadores.

Considera-se que o grau de intermediação avalia o quanto um pesquisador exerce papel de mediador ou está entre dois pesquisadores em um caminho mais curto, denominado "geodésico".

Tabela 2: Cálculo da centralidade de intermediação dos pesquisadores bolsistas de produtividade em pesquisa do $\mathrm{CNPq}$ em Ciência da Informação (2005-2009)

\begin{tabular}{lc}
\hline Pesquisadores & Betweenness \\
\hline M. Fujita & 53.0 \\
B. Cendón & 40.0 \\
J. de Moraes & 32.0 \\
J. Guimarães & 29.0 \\
M. Melo & 23.0 \\
R. Marteleto & 15.0
\end{tabular}




\begin{tabular}{lc} 
M. L. de A. Campos & 11.0 \\
E. Suaiden & 10.0 \\
L. V. P. Ribeiro & 6.0 \\
S. Mueller & 6.0 \\
C. Marcondes & 5.0 \\
I. Freire & 2.0 \\
R. N. M. dos Santos & 2.0 \\
S. Caregnato & 2.0 \\
G. Olinto & 1.0 \\
M. Tálamo & 1.0 \\
M. Valentim & 1.0 \\
S. Monteiro & 1.0 \\
T. Burnham & 1.0 \\
D. Población & 0.0 \\
E. Orrico & 0.0 \\
I. Thiesen & 0.0 \\
J. Jardim & 0.0 \\
M. A. Moura & 0.0 \\
M. Aquino & 0.0 \\
M. da Cunha & 0.0 \\
M. de Lara & 0.0 \\
M. González de Gómez & 0.0 \\
N. Kobashi & 0.0 \\
P. Costa Santos & 0.0 \\
R. F. de Souza & 0.0 \\
S. Albagli & 0.0 \\
S. G. Baptista & 0.0 \\
V. Dodebei & 0.0 \\
V. Pinto & 0.0 \\
Z. de Oliveira & 0.0 \\
\hline F. Dadoscatos \\
M
\end{tabular}

Fonte: Dados calculados pelo Software Ucinet.

A análise dos indicadores de centralidade de intermediação mostra que os 82 pesquisadores que tiveram grau de intermediação zero não fazem intermediação com nenhum outro. Nesse sentido, pode ocorrer intermediação quando o pesquisador possui traços relacionais com pelo menos dois outros pesquisadores os quais intermedeia. Por exemplo: dentro da rede de colaboração científica entre os pesquisadores (2005-2009), encontra-se uma pequena sub-rede formada pelos seguintes pesquisadores: I. Freire, G. Freire, V. Araújo e M. Miranda. Para melhor exemplificar o significado de centralidade de intermediação, tome-se, por exemplo, I. Freire, que possui, nos dados da Tabela 2, intermediação 2, ou seja, uma posição nesta pequena sub-rede, exercendo o papel de mediadora entre os demais pesquisadores pertencentes à sub-rede. Para se conectarem entre si, tem I. Freire como mediadora em dois caminhos possíveis.

M. Fujita faz intermediação com grande número de pesquisadores, o que pode ser comprovado por ter o maior valor de intermediação (betweenness centrality): 53.0.

B. Cendón também se destaca entre os pesquisadores, com alto poder de intermediação $(40,0)$, e apresenta uma parceria com a M. Oliveira, bolsista de produtividade em pesquisa do CNPq em CI.

Considerando os critérios utilizados para contextualizar o grau de centralidade citado, pode-se apresentar também a centralidade de intermediação, destacando que 19 pesquisadores bolsistas de produtividade em pesquisa do CNPq em CI, no período de 20052009, apresentaram valor de centralidade de intermediação.

\subsection{Indicadores da rede de colaboração científica, no período de 2010 a 2014}

Apresentam-se, na Tabela 3, os diferentes graus de centralidade, destacandose a pesquisadora $M$. Fujita com maior grau de centralidade - 25.0 -, significando que possui 25 ligações ou coautorias com os demais pesquisadores.

L. Bufrem, pesquisadora bolsista de produtividade em pesquisa do $\mathrm{CNPq}$ em $\mathrm{CI}$, possui o segundo maior grau de centralidade de grau - 17.0 -, significando que possui 17 ligações com os demais pesquisadores.

J. Guimarães, pesquisador bolsista de produtividade em pesquisa do $\mathrm{CNPq}$ em $\mathrm{CI}$, possui o terceiro maior grau de centralidade 15.0 -, significando que possui 15 ligações ou coautorias com os demais pesquisadores. 
Tabela 3: Cálculo do grau de centralidade dos pesquisadores bolsistas de produtividade em pesquisa do $\mathrm{CNPq}$ em Ciência da Informação (2010-2014)

\begin{tabular}{|c|c|}
\hline Pesquisadores & Degree \\
\hline M. Fujita & 25.0 \\
\hline L. Bufrem & 17.0 \\
\hline J. Guimarães & 15.0 \\
\hline M. Valentim & 13.0 \\
\hline B. Cendón & 12.0 \\
\hline M. Hayashi & 12.0 \\
\hline R. Souza & 11.0 \\
\hline R. N. M. dos Santos & 10.0 \\
\hline E. Suaiden & 9.0 \\
\hline M. Grácio & 9.0 \\
\hline S. Vidotti & 9.0 \\
\hline E. T. de Oliveira & 8.0 \\
\hline G. Dias & 8.0 \\
\hline I. Freire & 7.0 \\
\hline P. Costa Santos & 7.0 \\
\hline E. Ferneda & 6.0 \\
\hline M. L. de A. Campos & 6.0 \\
\hline M. Melo & 6.0 \\
\hline R. Marteleto & 6.0 \\
\hline H. Casarin & 5.0 \\
\hline M. Moura & 5.0 \\
\hline N. Kobashi & 5.0 \\
\hline S. M. S. P. Ferreira & 5.0 \\
\hline V. Pinto & 5.0 \\
\hline E. Orrico & 4.0 \\
\hline J. de Moraes & 4.0 \\
\hline M. Aquino & 4.0 \\
\hline S. Caregnato & 4.0 \\
\hline A. Lopez & 3.0 \\
\hline L. V. P. Ribeiro & 3.0 \\
\hline M. de Lara & 3.0 \\
\hline V. Dodebei & 3.0 \\
\hline C. Marcondes & 2.0 \\
\hline E. Murgia & 2.0 \\
\hline M. Tomaél & 2.0 \\
\hline N. Oddone & 2.0 \\
\hline S. Monteiro & 2.0 \\
\hline
\end{tabular}

G. Olinto

1.0

I. Thiesen $\quad 1.0$

J. Jardim $\quad 1.0$

M. Bräscher $\quad 1.0$

M. de Almeida $\quad 1.0$

M. S. de F. Gomes $\quad 1.0$

M. Tálamo $\quad 1.0$

R. F. de Souza $\quad 1.0$

S. Albagli $\quad 1.0$

S. Amaral 1.0

V. Beraquet $\quad 1.0$

Fonte: Dados calculados pelo Software Ucinet.

Observando o grau de centralidade dos pesquisadores bolsistas de produtividade em pesquisa do CNPq em CI (2010-2014), reforçase a afirmação de que alguns deles compõem o grupo de pesquisadores centrais ou periféricos da rede de colaboração científica.

Apresentam-se, na Tabela 4, as diferentes centralidades de intermediação. Considera - s e que o grau de intermediação avalia o quanto um pesquisador exerce papel de mediador ou está entre dois pesquisadores em um caminho mais curto - "geodésico".

A análise dos indicadores de centralidade de intermediação mostra que os 175 pesquisadores que tiveram grau de intermediação zero não fazem intermediação com nenhum outro. Nesse sentido, pode ocorrer intermediação quando o pesquisador possui traços relacionais com pelo menos dois outros pesquisadores os quais intermedeia, Por exemplo: dentro da rede de colaboração científica entre os pesquisadores (2010-2014), encontra-se uma pequena sub-rede formada pelos seguintes pesquisadores: R. Marteleto, M. Tomaél, V. Couzinet, S. Kovacs, Y. Maury, H. David; M. Bteshe e T. Zaninelli.

Para melhor exemplificar o significado de centralidade de intermediação, tome-se, por exemplo, R. Marteleto, que possui, nos dados na Tabela 4, intermediação 18.0, ou seja, ela possui uma posição nesta pequena sub-rede, exercendo o papel de mediadora entre os demais pesquisadores pertencentes à sub-rede. Para se conectarem entre si, tem R. Marteleto como mediadora em 18 caminhos possíveis. 
Tabela 4: Cálculo da centralidade de intermedição dos pesquisadores bolsistas de produtividade em pesquisa do CNPq em Ciência da Informação (2010-2014)

\begin{tabular}{|c|c|c|c|}
\hline \multirow{4}{*}{\multicolumn{2}{|c|}{$\begin{array}{l}\text { Tabela 4: Cálculo da centralidade de } \\
\text { intermedição dos pesquisadores bolsistas de } \\
\text { produtividade em pesquisa do CNPq em Ciência } \\
\text { da Informação (2010-2014) }\end{array}$}} & V. Dodebei & 0.5 \\
\hline & & C. Marcondes & 0.0 \\
\hline & & G. Olinto & 0.0 \\
\hline & & I. Thiesen & 0.0 \\
\hline Pesquisadores & Betweenness & J. Jardim & 0.0 \\
\hline M. Valentim & 4925.0 & M. Bräscher & 0.0 \\
\hline S. Vidotti & 4782.6 & M. de Almeida & 0.0 \\
\hline G. Dias & 3081.7 & M. S. de F. Gomes & 0.0 \\
\hline L. Bufrem & 2836.0 & M. Tálamo & 0.0 \\
\hline M. Fujita & 2696.0 & R. F. de Souza & 0.0 \\
\hline R. Souza & 2266.8 & S. Albagli & 0.0 \\
\hline M. Grácio & 1972.2 & S. Amaral & 0.0 \\
\hline E. T. de Oliveira & 1868.7 & V. Beraquet & 0.0 \\
\hline
\end{tabular}

J. Guimarães 1739.7

E. Ferneda 1051.9

B. Cendón $\quad 960.2$

R. N. M. dos Santos 911.0

P. Costa Santos $\quad 782.0$

I. Freire $\quad 529.8$

E. Suaiden $\quad 525.0$

V. Pinto 441.3

N. Kobashi $\quad 396.0$

H. Casarin $\quad 395.0$

J. de Moraes $\quad 170.2$

M. Melo 134.9

N. Oddone $\quad 133.0$

M. Hayashi $\quad 54.7$

R. Marteleto $\quad 18.0$

M. Moura $\quad 14.0$

S. M. S. P. Ferreira 8.0

M. Aquino $\quad 6.0$

M. Tomaél $\quad 6.0$

S. Monteiro $\quad 5.0$

S. Caregnato $\quad 4.0$

E. Orrico $\quad 3.5$

A. Lopez $\quad 3.0$

M. de Lara $\quad 3.0$

L. V. P. Ribeiro 2.0

E. Murgia $\quad 1.0$

M. L. de A. Campos $\quad 1.0$

M. Valentim também possui uma posição de destaque entre os pesquisadores, tendo alto poder de intermediação (4925.0), pois apresenta uma parceria com dois pesquisadores bolsistas de produtividade em pesquisa do $\mathrm{CNPq}$ em $\mathrm{CI}$ : E. T. de Oliveira e M. Fujita.

S. Vidotti faz intermediação com grande número de pesquisadores, o que pode ser comprovado por ter o maior valor de intermediação (betweenness centrality): 4782.6.

\subsection{Análise das redes sociais (ARS) e a sociologia de Pierre Bourdieu}

Em relação à ARS, especificamente os "Indicadores das redes de colaboração científica" - grau de centralidade (centrality degree) e centralidade de intermediação (betweenness centrality) -, no primeiro (2005-2009) e segundo quinquênios (2010-2014) calculados, analisados e relacionados (possiveis articulações) aos conceitos da Sociologia de Pierre Bourdieu, pode-se concluir o seguinte: possuir alto valor de grau de centralidade - no caso desta pesquisa, em relação aos demais pesquisadores bolsistas de produtividade em pesquisa do $\mathrm{CNPq}$ apresentados nas redes de colaboração científica - significa, para o Campo Científico da CI e suas singularidades, que ele é um agente mais ativo nessa estrutura social e com um significativo Capital Social que diz respeito à posse de uma rede durável de relações e/ou interações. 
Deve-se considerar os aspectos subjacentes ao espaço dinâmico de produção do conhecimento científico, isto é, que ele possui o maior número de publicações em coautoria, especialmente os artigos científicos, do que os outros pesquisadores bolsistas de produtividade em pesquisa do $\mathrm{CNPq}$ no Campo da CI.

$\mathrm{O}$ alto valor da centralidade de intermediação apresentada pelos pesquisadores bolsistas de produtividade em pesquisa do $\mathrm{CNPq}$ mostra que o agente está em uma posição (ou em postos) de destaque nesse espaço hieraquizado em comparação com os demais pesquisadores bolsistas de produtividade em pesquisa do $\mathrm{CNPq}$, considerando as alianças positivas ou negativas, tais como conflito, concorrência e/ ou cooperação. Leva-se em conta a rede de colaboração científica formada no Campo da $\mathrm{CI}$, na medida em que é responsável pelo compartilhamento de informação e conhecimento representado pelas ligações e/ou relações apresentadas nas redes de colaboração científica que não produzem um contato direto, ou seja, mensura a importância e o controle do agente na circulação da informação dentro de uma da rede de colaboração científica.

Ainda, representa a habilidade que ele possui em se relacionar com os coautores importantes do Campo em questão (FELLMAN; WRIGHT, 2008). Essas características da centralidade de intermediação podem ser relacionadas com o conceito de Capital Científico, especificamente o "institucional, temporal, político ou poder sobre o mundo científico", considerando os aspectos estratégicos que cada agente adota para ocupar uma posição de destaque no interior do Campo da CI.

Os pesquisadores bolsistas de produtividade em pesquisa do $\mathrm{CNPq}$ em $\mathrm{CI}$, que possuem valores mais altos, portanto representativos por meio das medidas de grau de centralidade e centralidade de intermediação, podem ser apresentados como os agentes dominantes no Campo da CI, considerando suas relações objetivas, tendo em vista as regras presentes nesse espaço estruturado de posições ocupadas por dominantes (conservadores), dominados (subversivos) e pretendentes (são os agentes que ainda não estão participando do jogo, ou seja, aqueles que ainda não estão inseridos em um espaço social de lutas, conflitos e concorrência). Isso mostra que há uma disputa constante pelas posições sociais no Campo da CI, no caso, das redes de colaboração científica (BOURDIEU, 1976).

As práticas de coautoria indicam que o grupo de pesquisadores bolsistas de produtividade em pesquisa do $\mathrm{CNPq}$ e seus coautores doutores no Campo da CI apresenta um Capital Social representativo, que pode influenciar na posição na rede de colaboração científica, ou seja, disputa, ao longo dos anos, posições centrais na estrutura social do Campo Científico. Isso sugere que há uma luta significativa pelas posições de maior destaque na rede de colaboração científica, uma luta na qual os agentes utilizam estratégias sociais pautadas pelas aceitação e pela adequação à hierarquia presente nesse contexto com o intuito de acumular Capital Científico do tipo puro, tais como reconhecimento por meio de citações, e do tipo institucional, tais como cargos, e, consequentemente, galgar e (ou) manter posições de destaque (LIMA, 2011).

Tais práticas mostram que existe uma estrutura social de relações e de conectividade que pode se manter ao longo do tempo e dos anos nas redes de colaboração científica. A reincidência constatada indica a existência de um habitus de grupo (BOURDIEU, 1996), que contribui para uma tendência de reprodução social da estrutura de parcerias na produção do conhecimento científico e na dinâmica e evolução das redes de colaboração científica entre os pesquisadores bolsistas de produtividade em pesquisa do CNPq e seus coautores doutores no Campo da CI (LIMA, 2011).

\section{CONSIDERAÇÕES FINAIS}

Nas considerações finais, desenvolve-se uma análise sistematizada baseada nos resultados coletados, organizados e fundamentados para responder às questões apresentadas na introdução deste artigo científico.

Assim, responde-se à seguinte questão: como esses pesquisadores se colocam no Campo da CI e qual posição eles ocupam no Campo em estudo?

Em relação à análise dos Grafos que se referem à rede de colaboração científica (2005 a 2009 e 2010 a 2014), respectivamente, identificouse que, no período de 2005 a 2009, havia muitos 
subcampos isolados e poucos articulados entre si no interior do Campo da CI, além de uma frágil internacionalização com as parcerias estrangeiras. Ainda, no período de 2010 a 2014, a rede de colaboração científica se apresenta com maior interconexão entre os pesquisadores bolsistas de produtividade em pesquisa do $\mathrm{CNPq}$ em CI, com aspectos significativos das parcerias internacionais, mostrando o fortalecimento do Campo e de sua identidade em âmbito mundial.

A partir dessas questões, ratifica-se que o Campo é um espaço de lutas sociais e científicas, pois sua estrutura é definida pela distribuição desigual do Capital. Neste caso, refere-se ao Capital Social, que está ligado ao número de coautorias, em âmbito nacional e/ou internacional, que os pesquisadores possuem no interior do Campo em estudo.

Considera-se que a proposta de fundamentar a ARS a partir de alguns conceitos da Sociologia de Pierre Bourdieu, especialmente a teoria do Campo, pode melhor elucidar os fundamentos das coautorias, bem como o número de artigos publicados, número de citações que os artigos receberam, número de coautores na rede de colaboração científica e cargos e/ou funções ocupadas no cenário acadêmico e científico.

De acordo com os fundamentos desta pesquisa e da Sociologia de Pierre Bourdieu, para ocupar uma posição de dominante, o pesquisador bolsista de produtividade em pesquisa do $\mathrm{CNPq}$ deve entender a lógica do jogo, pois o Campo possui eixos estruturantes a partir dos quais pode identificar os principais jogadores que ocupam esse espaço social de concorrência científica. É fundamental que o pesquisador adote estratégias sociais e políticas de acordo com as dinâmicas do Campo para acumular Capital Social e Capital Científico do tipo objetivado, puro e institucional, para almejar ou fazer a manutenção de sua posição social.

Com os subsídios já sistematizados, responde-se à seguinte questão: como explicar os mecanismos que fazem com que os pesquisadores passem a ocupar posições de dominantes, dominados e pretendentes no Campo da CI?

Torna-se um desafio compreender como a Sociologia de Pierre Bourdieu impregna as relações de construção da ciência como um todo, por meio de um processo de luta e oposições, competições e contrapontos.

Como recomendações para o prosseguimento desta pesquisa, destacase a relevância de se entender e analisar os mecanismos sociais que permitem aos pesquisadores bolsistas de produtividade em pesquisa do $\mathrm{CNPq}$ em $\mathrm{CI}$ ocupar posições de centro nas redes de colaboração científica.

Artigo recebido em II/07/20I8 e aceito para publicação em 0I/09/20I8

\section{SOME CONCEPTS OF THE SOCIOLOGY OF PIERRE BOURDIEU RELATED TO SOCIAL NETWORK ANALYSIS}

ABSTRACT Information Science (IS) is a hierarchical field, relatively autonomous and formed by objective relations that occur in a social context. This research aimed to understand the implicit mechanisms in the knowledge generation and construction of the Information Science CNPq (National Council of Scientific and Technological Development) fellow researchers, and the social position they occupy in the field. More specifically, we sought to understand the social mechanisms built to organize and which facilitate the structuring of the scientific collaboration networks of these CNPq researchers in IS; to underpin the Social Network Analysis (SNA) from the Field Theory and the concepts of Social Capital and Science Capital, proposed by Pierre Bourdieu's Sociology. For constructing the scientific collaboration networks among the researchers, Ucinet Software was used. The Science Field theory was also used to complement the Social Network Analysis procedure, focusing on the characterization and evolution of scientific collaboration networks (2005-2009 and 2010-2014). In conclusion, according to the Sociology of Pierre Bourdieu, to occupy a dominant position, the CNPq fellow researcher must understand the logic of the practice, since the field has structuring axes from which the main players occupying this social space of scientific competition are identified.

Keywords: $\quad$ The Sociology of Pierre Bourdieu. Social Network Analysis. Information Science. 


\section{REFERÊNCIAS}

BEN-DAVID, J. Introdução. In:__. Sociologia da Ciência. Tradução Newton T. Gonçalves. Rio de Janeiro: FGV, 1975. p.1-32.

BOURDIEU, P. Coisas Ditas. Tradução Cássia R. da Silveira e Denise Moreno Pegorim; revisão técnica Paula Montero - São Paulo: Brasiliense, 2004a.

Le capital social: notes provisoires. Actes de la recherche en sciences sociales, v. 1, p.2-3, 1980.

Le champ scientifique. Actes de la Recherche em Sciences Sociales, v. 2, n. 2/3, p. 88-104, 1976.

Os usos sociais da ciências: por uma sociologia clínica do campo científico. São Paulo: UNESP, 2004b.

Para uma sociologia da ciência. Lisboa: Ed. 70, 2008.

Questões de sociologia. Rio de Janeiro: Marco Zero, 1983.

Razões práticas. Campinas: Papirus, 1996.

BOURDIEU, P.; CHAMBOREDON, J.C.; PASSERON, J.-C. Ofício de sociólogo: metodologia da pesquisa na sociologia. Tradução de Guilherme João de Freitas Teixeira. 8. ed. Petrópolis: Vozes, 2015.
FELLMAN, P. V., WRIGHT, R., Modelando redes terroristas. In: DUARTE, F.; QUANDT, C.; SOUZA, Q. (Orgs.). O tempo das redes. Perspectiva. 2008. p.259.

HAYASHI, C. R. M. et al. Análise de redes de colaboração científica entre educação especial e fonoaudiologia. Revista Interamericana de Biblioteconologia, v.35, n.3, p.285-297, 2012.

JOHNSON, C. T. el at. Social Capital and Information Science Research. Information Research, v.20, n.4, p.1-6, 2008.

LIMA, M. Y. Coautoria na produção científica do PPGGeo/UFRGS: uma análise de redes sociais. Ciência da Informação, v.40, n.1, p.3851, 2011.

MARTINS, D. L. Análise de redes sociais de colaboração científica no ambiente de uma federação de bibliotecas digitais. 2012. 256f. Tese (Doutorado em Ciência da Informação ) - Escola de Comunicação e Artes/USP, Universidade de São Paulo, São Paulo, 2012.

MOREIRA JR., A.; F.; ANDRADE, T. H. N. de. Pierre Bourdieu e a Noção de Campo Científico: contribuições para o estudo da prática científica e técnica. In: HAYASHI, M. C. P. I.; RIGOLIN, C. C. D.; KERBAUY, M. T. M. (Orgs.). Sociologia da Ciência: contribuições ao campo CTS. Campinas: Editora Alínea, 2014. p.161-181.

NEWMAN, M., BARABASI, A., WATTS, D. The structure and dynamics of networks. Princeton University Press, 2006.

OTTE, E.; ROUSSEAU, R. Social network analysis: a powerful strategy, also for the 
Information sciences. Journal of Information

Science, v.28, n.6, p.441-453, 2002.

PINHEIRO, L. V. R. A Ciência da Informação entre sombra e luz: domínio epistemológico e campo interdisciplinar. 1997. 266 f. Tese (Doutorado em Comunicação) - Escola de Comunicação, Universidade Federal do Rio de Janeiro, Rio de Janeiro, 1997. 